\title{
Modulation of L-Arginine-Arginase Metabolic Pathway Enzymes: Immunocytochemistry and mRNA Expression in Peripheral Blood and Tissue Levels in Head and Neck Squamous Cell Carcinomas in North East India
}

\author{
Shilpee Srivastava, Sankar Kumar Ghosh*
}

\begin{abstract}
Background: Arginine may play important roles in tumor progression by providing ornithine for polyamine biosynthesis, required for cell growth. The aim of this work was to determine the expression of arginine metabolic pathway enzymes in head and neck squamous cell carcinoma (HNSCC) in northeast India. Materials and Methods: The expressions of arginase isoforms (ARG1 and ARG2), ornithine aminotransferase (OAT) and ornithine decarboxylase (ODC) were examined in fifty paired HNSCC and adjacent non-tumor tissues by immunohistochemistry. Immunocytochemistry, semiquantitative reverse transcription sq-PCR and quantitative real-time qPCR were used to assess protein and mRNA expressions in peripheral blood of fifty HNSCC patients and hundred controls. Results: ARG1 and ODC protein and mRNA were strongly expressed in peripheral blood from HNSCC patients. No ARG2 expression was observed. In vivo, expression of ARG1, ARG2 and ODC was significantly higher in tumor than in non-tumor tissues. Most tumors expressed low levels of OAT, with no difference in tissues or blood, compared to controls. The absolute extent of maximal ARG1 upregulation with qPCR showed 6.23 fold increase in HNSCC. Conclusions: These findings strongly suggest that in HNSCCs, the ARG1 pathway is stimulated leading to the formation of polyamines as indicated by higher ODC expression, which promote tumor growth.
\end{abstract}

Keywords: HNSCC - arginase - biomarker - ornithine decarboxylase - arginine metabolism

Asian Pac J Cancer Prev, 16 (16), 7031-7038

\section{Introduction}

Head and neck squamous cell carcinoma (HNSCC) is the fifth most common cancer worldwide and is the most common neoplasm in central Asia (Hashibe et al., 2009). Although early-stage HNSCC has high cure rates, up to $50 \%$ of patients present in advanced disease (Gourin and Podolsky, 2006). Among these advanced stages HNSCC patients, $60-80 \%$ will die within five years after diagnosis (Hauswald et al., 2011). In India, the HNSCC accounts for $30-40 \%$ cancers at all sites. It is the sixth most common cause of death in males and seventh in females. The cigarette smoking and tobacco chewing habits are highly prevalent among head and neck cancer patients. Tobacco is considered the single most frequent cause of head and neck cancer (Boffetta et al., 2008; Dal Maso et al., 2015). In Northeast India; incidence of tobacco related oral cancers is about 33\% (Bhattacharjee et al., 2006). Though, the prognosis of cancer has improved significantly over the past decades, HNSCC still remains one of the most common tumors worldwide. Therefore, a better understanding of the biology of this common tumor is necessary to improve both diagnosis and treatment.

Arginine is a conditionally essential amino acid necessary for growth in children and under some pathological conditions in adults. It is a substrate for protein synthesis and has numerous roles in cellular metabolism. It serves as an intermediate in the urea cycle and as a precursor for nitric oxide (NO), creatine and agmatine biosynthesis. Arginine is also a source of ornithine, a substrate for the biosynthesis of polyamines, glutamate and proline. Polyamines play a crucial role in many cancers, and its depletion leads to tumor regression in oral squamous cell carcinoma (Skorupski et al., 2011). Arginine levels are regulated by arginine hydrolyzing enzyme arginase (ARG). We earlier reported that serum ARG in combination with C-reactive protein (CRP) can effectively diagnose HNSCC (Choudhury et al., 2014). ARG catalyzes the hydrolysis of arginine to ornithine and urea. It is a key enzyme playing role in several pathways of intermediary metabolism. Two isoenzymes of ARG are known, ARG1 and ARG2. ARG isoforms 
provide a supply of ornithine, a crucial metabolite in the biosynthesis of polyamines in cells. Humans produce two $\mathrm{Mn}^{2}+$-dependent ARG isoenzymes (EC 3.5.3.1). ARG1 is highly expressed in the cytosol of hepatocytes and functions in nitrogen removal in the final step of the urea cycle. The ARG2 is localized in the mitochondria in all the tissues where it provides supply of ornithine for the polyamine biosynthesis (Lopez et al., 2005). Ornithine aminotransfearse (OAT; EC 2.6.1.13) catalyses the conversion of L-ornithine and a 2-oxo acid to L-glutamate 5-semialdehyde and an L-amino acid. It is present in mitochondrial matrix and controls the level of L-ornithine in cells and tissues. Ornithine decarboxylase (ODC; EC 4.1.1.17) is a pyridoxal-5' phosphate dependent enzyme. It is a cytosolic enzyme catalyzes the decarboxylation of L-ornithine to putrescine, a rate limiting to steps to step in the polyamine biosynthesis.

Long-term studies showed that ARG activity is raised in blood serum of cancer patients. However, serum ARG activity can be increased in some non-malignant disease such as type two diabetes mellitus, asthma, sickle-cell anemia, burn or pancreatitis (Biczo et al., 2010; Yamamoto et al., 2012; Ramirez-Zamora et al., 2013; Barron et al., 2013). An increase in ARG1 and ODC immunoreactivity was observed in smoking compared with nonsmoking asthmatic patients (Bergeron et al., 2007). Tobacco specific nitrosamines are carcinogenic in animals and reported to increase ODC activity in embryonic mouse tongue epithelial cells (Gijare et al., 1989). Oxidative damage caused by smoking results in the up regulation of ARG pathway and induction of both ARG and OAT activities (Matthaei and Williams, 1987; Yamamoto et al., 2012). The fate of ornithine depends on the activity of the mitochondrial ornithine transporter. The ornithine which remains in the mitochondrial matrix is acted upon by OAT to form glutamine whereas, the ornithine which is transported out of the mitochondria is acted upon by the cytosolic enzyme ODC to form polyamines, through the cascade of reactions. Therefore, the aim of this study was to examine OAT and ODC expression in addition to ARG isoenzymes to find out the possible mechanism of tumor growth. Besides, their expression can be used as biomarker to diagnose HNSCC. The expression levels were determined in the peripheral blood and tissues of human head and neck cancer patients using immunocytochemistry, semi-quantitative reverse transcription polymerase chain reaction (sqPCR), quantitative real-time RT-PCR (qPCR) and immunohistochemistry.

\section{Materials and Methods}

Patients: This study included 50 patients. Hundred healthy blood donors were included as control individuals. All patients provided informed written consent before inclusion. The study was approved by the Ethical Review Board at Assam University Silchar, India. Blood samples were taken before surgery in ethylene diamine tetra acetic acid (EDTA) vial, and a part was collected in trizol and stored at $-80^{\circ} \mathrm{C}$. Formalin fixed paraffin embedded (FFPE) tissue sections of same patients were obtained from local biobank. All cases were histologically reviewed and confirmed of high grade HNSCC. Adjacent normal tissues were taken as controls.

Immunocytochemistry: Peripheral blood smears were prepared on a clean poly L-lysin coated microscopic slides. Slides were air-dried overnight at room temperature. The preparation was fixed with acetone $\left(-18^{\circ} \mathrm{C}\right)$ for 5 minutes. The inhibition of endogenous peroxidase activity was performed with hydrogen peroxide $\left(\mathrm{H}_{2} \mathrm{O}_{2}\right)$-methanol solution for 30 minutes at $37^{\circ} \mathrm{C}$. The slides were washed three times in distilled water. The blocking of non-specific binding was done using phosphate buffer saline (PBS) containing $1 \%$ bovine serum albumin (BSA) for $2 \mathrm{~h}$ at room temperature. Cells were stained with appropriate primary antibody (Table 1 ) at $4^{\circ} \mathrm{C}$, overnight in a humid chamber. After washing three times with PBS, horseradish peroxidase (HRP) conjugated secondary antibody was applied at room temperature for $2 \mathrm{~h}$. After washing three times, 3, 3'-diaminobenzidine (DAB) was added for 10 min. A brown product was formed by the reaction of peroxidase with DAB (Erber et al., 1984; Molnar et al., 2001). All slides were examined by two experienced observers, and an estimate of positive cells was made by counting 100-200 cells in a representative section on the slide.

Immunohistochemistry: Routinely processed, FFPE tissue specimens obtained from biopsies and/or surgical resection of tumors in all 50 cases. FFPE tissue block was chosen based on the haematoxylin, and eosin stained slides. Immunohistochemical staining was performed using DAB-HRP method. FFPE tissues were cut into $5 \mu \mathrm{M}$ sections. Glass mounted tissue sections were deparaffinized in xylene and were rehydrated in decreasing concentrations of ethanol, then washed in distilled water. Slides were immersed in $\mathrm{H}_{2} \mathrm{O}_{2}$-methanol solution to inhibit any endogenous peroxidase activity. Subsequently, sections were subjected to heat induced epitope retrieval by heating in a microwave oven in a $10 \mathrm{mM}$ citrate buffer (pH 7.0) for 10 minutes (evaporated buffer was replaced in between). Immunostaining was performed as described earlier. Slides were counterstained with hematoxylin.

Tumor cells were considered positive if definite staining distant from edges of the section or necrosis was observed. The extent of immunostaining in each specimen was observed by two independent observers without knowledge of the patients' clinical details. Staining of tumor and normal tissue, from a single slide, was evaluated by a semiquantitative scoring system for intensity and extent of positive cells. The system assesses the staining intensity as a 4-level ordered categorical variable ( 0 , none; 1 , mild; 2 , moderate; and 3 , strong), and the percentage of positive cells are assessed as a 5-level ordered categorical variable ( 0 , none or rare cells; $1,10 \%$; $2,10-25 \% ; 3,25-50 \%$; and $4,>50 \%$ ). Case tumors were considered positive if the immunohistochemical staining to detect expression of ARG1 protein had an intensity score of moderate or strong, had at least $10 \%$ or more of cells showing evidence of expression, and was considered positive by both study pathologists. The rationale for the cutoff point was based on the background level of the normal adjacent tissue on the tumor sections; tumor tissue that showed staining below this threshold was considered 
negative for protein expression by immunohistochemical detection. Appropriate positive and negative (staining lacking primary antibodies) controls were used in each batch of staining.

RNA extraction and sqPCR: We also performed sqPCR analysis for the expression of the ARG1,ARG2, ODC and OAT genes on blood samples available from all 50 cases. Total RNA was extracted from blood using TRIzol reagent (Invitrogen) according to the manufacturer's instructions, and treated with RNase-free DNase. 1-2 $\mu \mathrm{g}$ total RNA was used to synthesize the first strand cDNA using SuperScript RT-PCR kit (Invitrogen). Both RNAand cDNA concentrations were determined spectrophotometrically (Biorad Spectronanodrop). The primer sequences included sense 5'-ATTGTTCCGTTCTTCTTGACTT-3' and antisense 5'-AGTGTGATGTGAAGGATTATG-3' for ARG1 (148bp), sense 5'-GGTCCCGCTGCCATAAGAG-3' and anti-sense 5'-CGTGGATTCACTATCAGGTTGTTG-3' f o r A R G 2 ( 137 b p $), \quad$ s e n s e 5'-GGATCCAAATGTGGCTGCGTTCAT-3' and antisense 5'-AACAGCCAGCCATCTACCAGTTCT-3' f o r O A T ( $175 \mathrm{~b} \mathrm{p}), \quad$ s e n s e 5'-GTGGGTGATTGGATGCTCTTTG-3' and anti-sense 5' - AGGCCCTGACATCACATAGTAG-3' for ODC (108bp), sense 5'- GAAATCCCATCACCATCTTCC-3' and anti-sense 5'-GAGCCCCAGCCTTCTCCATG - 3) for GAPDH (120bp), and sense 5'-TGGATCAGCAAGCAGGAGTATG-3' and antisense 5'-AAGAAAGGGTGTAACGCAACTAAG-3' for $\beta$-actin (98bp) (Bergeron et al., 2007; Wolf et al., 2007; Haenisch et al., 2008; Kitowska et al., 2008; Kim et al., 2012; Mondal et al., 2013). For all analysis, sqPCR assays were performed in a $25 \mu \mathrm{L}$ system containing PCR master mix, 30ng first-strand cDNA template and $0.4 \mu \mathrm{M}$ appropriate primers.

The PCR conditions were as follows: $94^{\circ} \mathrm{C}$ for 5 min, 27-34 cycles of $94^{\circ} \mathrm{C}$ for $30 \mathrm{~s}$, annealing for $30 \mathrm{~s}$, $72^{\circ} \mathrm{C}$ for $30 \mathrm{~s}$, and a last run at $72^{\circ} \mathrm{C}$ for $10 \mathrm{~min}$. Gene expression levels were quantified as band fluorescence intensity on a $2 \%$ agarose gel after electrophoresis using 1D image analysis software (Scientific imaging systems). Band intensities were normalized to the glyceraldehyde 3-phosphate dehydrogenase (GAPDH) transcript band for relative quantification.

\section{Real-time qPCR}

The StepOne ${ }^{\mathrm{TM}}$ Real-Time PCR System (Applied Biosystems) was used to perform PCR amplification for ARG1 cDNA. $\beta$-actin was used as a 'housekeeping gene' to normalize all of the threshold cycle $(\mathrm{Ct})$ values. The qPCR was performed in a final volume of $10 \mu \mathrm{l}$ with $0.5 \mu \mathrm{l}$ of template cDNA at a concentration of $20 \mathrm{ng} / \mu \mathrm{l}$ with $5 \mu \mathrm{l}$
SYBR green I fluorescent dye and $10 \mathrm{pM}$ of each primer for the target gene and the $\beta$-actin gene. The real-time PCR conditions consisted of initial denaturation and Taq polymerase activation at $95^{\circ} \mathrm{C}$ for 4 minutes followed by 40 cycles of $95^{\circ} \mathrm{C}$ for 45 seconds, $58^{\circ} \mathrm{C}$ for 1 minute, and $72^{\circ} \mathrm{C}$ for 1 minute and followed by a melting curve analysis. Each measurement was repeated in triplicate and a non-template control was included in each experiment. Results of real-time PCR were represented as Ct values, where $\mathrm{Ct}$ was a fraction defined as the cycle number at which the sample fluorescent signal passes a given threshold above the baseline. $\Delta \mathrm{Ct}$ was the difference in the $\mathrm{Ct}$ values derived from the ARG1 gene after being assayed and $\beta$-actin. The $\mathrm{N}$-fold differential expression in ARG1 gene of a tumor sample and matched normal tissue were expressed as $2^{\Delta \mathrm{Ct}}$. The significance level was defined as a p-value of less than 0.01 .

\section{Results}

Patients: All the patients were scheduled to undergo surgical tumor resection. None had received preoperative therapy. Details of the patient population and their clinical characteristics are summarized in Table 2 and Figure 1. The mean age of study populations was 50 years, approximately $75 \%$ were males and $25 \%$ females. All the HNSCC patients included in the study were consuming either betel nut, smokeless tobacco, or smoking, or the combination of these three.

Among healthy subjects, $53 \%$ were without any carcinogenic habits, whereas, $9 \%, 6 \%, 26 \%, 2 \%$ and $4 \%$ were having betel nut, betel nut with smokeless tobacco, smokeless tobacco Smokeless tobacco with smoking and smoking habits, respectively. Among patients, $8 \%, 4 \%$ and $8 \%$ were habitual to betel nut, smokeless tobacco chewers or smokers, respectively. $24 \%$ and $56 \%$ patients used to consume betel nut with smokeless tobacco and smokeless tobacco with smoking, respectively. Primary cancer sites included larynx, oral cavity, NHL neck, retromolar, esophagus, epiglottis and cheek.

Blood samples from patients were drawn before surgery. Adjacent normal tissue from resected tumors and healthy donor's blood were enrolled as controls.

Expression of ARG isoforms, ODC and OAT in peripheral blood of HNSCC patients: We observed a trend towards a higher frequency of ARG1 and ODC expressing cells in peripheral blood from cancer patients compared with those from healthy donors $(p<0.0001)$. Examples of immunostaining are given in Figure 2 and 3. All in all $94 \%$ HNSCC patients were found to be positive for ARG1, whereas only $20 \%$ were positive among healthy donors. No expression of ARG2 protein was observed in

Table 1. Antibodies Used in the Study Indicating Clone, Source, Dilution, Visualization Method, Scoring, Threshold and Positive Controls

\begin{tabular}{|c|c|c|c|c|c|}
\hline Protein & Clone & Source & Dilution & Visualization & Positive Control \\
\hline ARG1 & Polyclonal & Santa Cruz & $1: 250$ & DAB & Liver \\
\hline ARG2 & Polyclonal & Santa Cruz & $1: 250$ & DAB & Kidney \\
\hline OAT & E.coli & Abcam & $1: 200$ & DAB & Liver \\
\hline ODC & HEK293T & Abcam & $1: 200$ & DAB & \\
\hline
\end{tabular}


Shilpee Srivastava and Sankar Kumar Ghosh

peripheral blood smears (Figure 2A). $92 \%$ of HNSCC patients were positive for ODC statining as compared to only $8 \%$ in healthy subjects. The differences were suggestive and statistically significant. Cells were positive for OAT, but most of them expressed only low levels of enzymes (Figure 3A). We did not detect any difference in the expression of OAT expression in HNSCC patients compared to control. Therefore, they were not account for any statistically significant difference between patients and controls, making OAT a minor contributor to tumor progression. Immunocytochemistry results were further confirmed by sqPCR.

The mRNA expression profiles showed ARG1 and ODC mRNAs upregulation in HNSCC (Figure 2B and 3B). Similar to protein expression results, we could not identify ARG2 mRNA in blood (Figure 2B). This is consistent with the recent finding that ARG2 is absent in blood circulation (Yang et al., 2013). Although, significant amount of OAT mRNA was present in both patients and controls but showed no significant difference (Figure 3B).

Expression of ARG isoforms, ODC and OAT in HNSCC tissues: To examine a possible relationship between expression in tissue and peripheral blood for

(A)
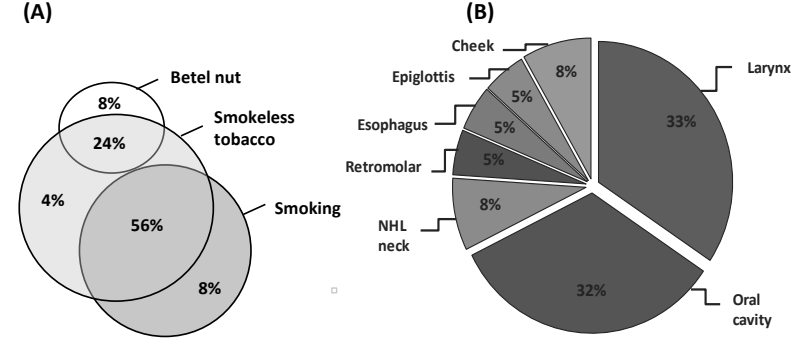

Figure 1. Figure 1. (A) Overlap of three habits, betel nut, tobacco chewing, and smoking in fifty head and neck squamous cell carcinoma patients. (B) Distribution of primary sites in head and neck squamous cell carcinoma patients. the proteins ARG1, ARG2, ODC and OAT, fifty FFPE HNSCC tissue samples were additionally stained with the respective antibodies (Figure $2 \mathrm{C}$ and $3 \mathrm{C}$ ). In vivo expression of ARG1 and ARG2 were more than two times higher in HNSCC tissue as compared to adjacent normal tissues (Table 3 ). The differences were statistically significant $(\mathrm{p}<0.0001)$. Higher ODC mRNA was also

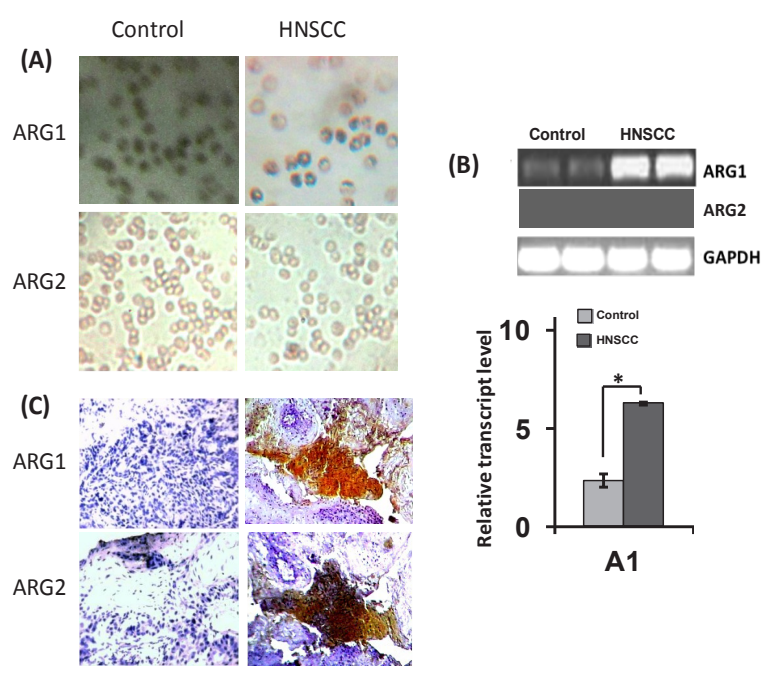

Figure 2. (A) Immunocytochemical patterns of blood cells of head and neck squamous cell carcinoma. Positive staining for ARG1 in HNSCC patients and, negative in controls. ARG2 is negative in both HNSCC and controls. (B) Semi-quantitative densitometry analysis of expression profile of ARG1 gene mRNA level. The values are mean of three independent experiments. The values are normalized to respective GAPDH values. Expression of ARG1, ARG2 and GAPDH genes mRNA in serum of HNSCC patients and controls. *represents that differences were statistically significant. (C) Immunohistochemical pattern of HNSCC. Positive staining for ARG1 and ARG2 in HNSCC and negative for controls. 1

Table 2. Characteristics of Head and Neck Squamous Cell Carcinoma Patients and Clinical Outcomes

\begin{tabular}{|c|c|c|c|}
\hline Variables & Control $(n=100)$ & Cases $(n=50)$ & $\mathrm{p}$ value \\
\hline Age in years $($ Mean \pm SD) & $50.91 \pm 11.55$ & $52.14 \pm 11.62$ & NS \\
\hline Gender & & & NS \\
\hline Male $(\%)$ & $75(75)$ & $70(35)$ & \\
\hline Female $(\%)$ & $25(25)$ & $30(15)$ & \\
\hline \multicolumn{4}{|l|}{ Habits } \\
\hline None & $53(53)$ & 0 & \\
\hline Betel nut (\%) & $9(9)$ & $8(4)$ & NS \\
\hline Betel nut + Smokeless Tobacco $(\%)$ & $6(6)$ & $24(12)$ & \\
\hline Smokeless Tobacco $(\%)$ & $26(26)$ & $4(2)$ & $<0.001$ \\
\hline Smokeless Tobacco $(\%)+$ Smoking $(\%)$ & $2(2)$ & $56(28)$ & \\
\hline Smoking $(\%)$ & $4(4)$ & $8(4)$ & $<0.001$ \\
\hline \multicolumn{4}{|l|}{ Cancer sites } \\
\hline Larynx & 0 & $33(17)$ & \\
\hline Oral cavity & 0 & 32 (16) & \\
\hline NHL neck & 0 & $8(4)$ & \\
\hline Retromolar & 0 & $5(3)$ & \\
\hline Esophagus & 0 & $5(3)$ & \\
\hline Epiglottis & 0 & $5(3)$ & \\
\hline Cheek & 0 & $8(4)$ & \\
\hline
\end{tabular}


observed in patients compared to controls $(\mathrm{p}<0.005)$.

Although differences in staining intensity could be observed by immunohistochemistry, it was difficult to correlate the intensities with blood mRNA expression levels. Most tumor tissues expressed very low levels of OAT enzyme protein, and we did not observe any significant difference in intensities between tumor and normal tissues $(\mathrm{p}<0.05)$.

ARG1 is frequently up-regulated in HNSCCs: Our sqPCR data indicated, mRNA level of ARG was increased remarkably in HNSCC as compared to healthy controls. To further validate whether ARG1 contributes to HNSCC progression, $\mathrm{qPCR}$ was performed to investigate mRNA levels of ARG1 in 50 HNSCC blood samples. As shown in

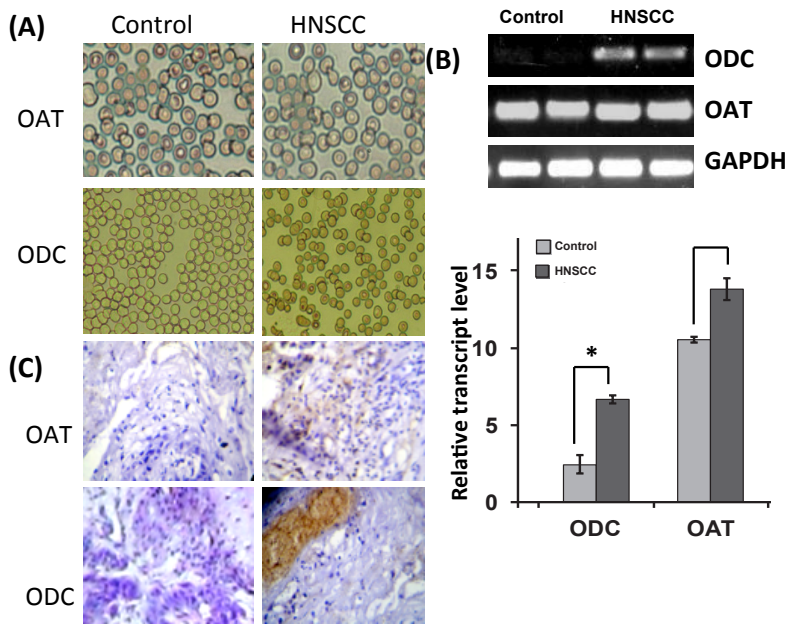

Figure 3. (A) Immunocytochemical pattern of blood cells of head and neck squamous cell carcinoma. Weak positive staining for OAT in HNSCC patients and controls. ODC is negative in controls and strongly positive in HNSCC and (B) Semi-quantitative densitometry analysis of expression profile of OAT, ODC genes mRNA level. The values are mean of three independent experiments. The values are normalized to respective GAPDH values. Expression of OAT, ODC and GAPDH genes mRNA in serum of HNSCC patients and controls. * represents that differences were statistically significant. (C) Immunohistochemical pattern of HNSCC. Weak positive staining for OAT in HNSCC and controls. Positive staining for ODC in HNSCC and negative for controls.
Figure 4A and 4B, ARG1 transcript levels in the HNSCC were significantly higher than in the controls $(p<0.01)$. The absolute extent of maximal upregulation depended on the technique used, with sqPCR giving 2 folds (Figure 2B)
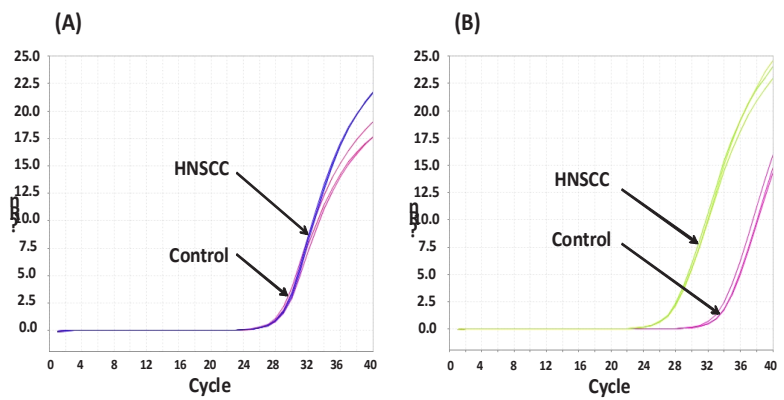

Figure 4. qPCR analysis of ARG1 mRNA. A) $\beta$-actin cDNA amplification plot of patient and healthy control subjects. B) ARG1 cDNA amplification plot of patient and healthy control subjects showing higher levels of expression in patients

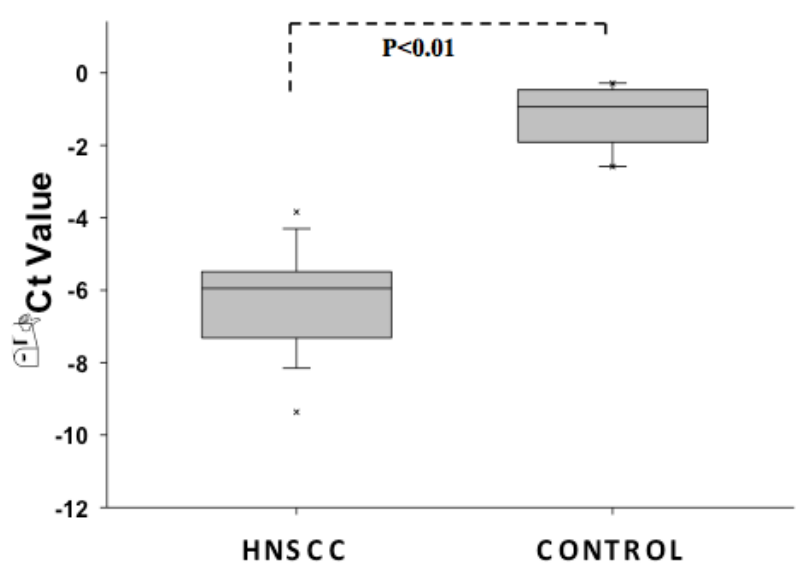

Figure 5. Expression of ARG1 mRNA in HNSCC using qPCR analysis with normal human blood as positive control, and no cDNA template as a negative control. For each sample, the relative mRNA level of ARG1 was normalized to $\beta$-actin. The line within each box represents the median $\mathrm{Ct}$ value; the upper and lower edges of each box represent the 75th and 25th percentiles, respectively; the upper and lower bars indicate the highest and lowest values determined, respectively.

Table 3. Contingency Analysis (Fisher's Exact Test) Comparing the Frequency of Protein expression in Control and HNSCC Patients Blood and Tissue

\begin{tabular}{|c|c|c|c|c|c|c|c|c|c|c|}
\hline \multirow{3}{*}{ Protein } & \multicolumn{5}{|c|}{ Immunocytochemistry in blood smear } & \multicolumn{5}{|c|}{ Immunohistochemistry in FFPE tissue } \\
\hline & \multicolumn{2}{|c|}{$\begin{array}{l}\text { Healthy } \\
\text { Control }\end{array}$} & \multicolumn{2}{|c|}{ HNSCC } & \multirow{2}{*}{$\begin{array}{c}\text { Control/ } \\
\text { HNSCC } \\
p(\mathrm{C} / \mathrm{H})\end{array}$} & \multicolumn{2}{|c|}{$\begin{array}{c}\text { Adjacent } \\
\text { Control }\end{array}$} & \multicolumn{2}{|c|}{ HNSCC } & \multirow{2}{*}{$\begin{array}{l}\text { Adjecent } \\
\text { Control/ } \\
\text { HNSCC } \\
\mathrm{p}(\mathrm{AC} / \mathrm{H})\end{array}$} \\
\hline & +ve/total & $\%(+)$ & +ve/total & $\%(+)$ & & +ve/total & $\%(+)$ & +ve/total & $\%(+)$ & \\
\hline ARG1 & $20 / 100$ & $20 \%$ & $47 / 50$ & $94 \%$ & $<0.0001$ & $20 / 50$ & $40 \%$ & $42 / 50$ & $84 \%$ & $<0.0001$ \\
\hline ARG2 & $0 / 100$ & $0 \%$ & $0 / 50$ & $0 \%$ & NS & $15 / 50$ & $30 \%$ & $39 / 50$ & $78 \%$ & $<0.0001$ \\
\hline OAT & $30 / 100$ & $30 \%$ & $15 / 50$ & $30 \%$ & NS & $23 / 50$ & $46 \%$ & $28 / 50$ & $56 \%$ & $<0.05$ \\
\hline ODC & $8 / 100$ & $8 \%$ & $46 / 50$ & $92 \%$ & $<0.0001$ & $21 / 50$ & $42 \%$ & $36 / 50$ & $72 \%$ & $<0.005$ \\
\hline
\end{tabular}


but with qPCR giving 6.23 fold increase in ARG1 mRNA levels as compared to healthy controls ( $p<0.01$, Figure 5). We got similar results using either $\beta$-actin or GAPDH as endogenous controls. This probably reflected difference in the semi-quantitative nature of densitometric analysis, and qPCR being more sensitive reproducible method to distinguish differences in gene expression.

We investigated the expression of these enzymes in relation to other clinical parameters, such as tumor grade, site, metastatic status, tumor size or habits. However, the intensity of ARG isoenzymes or ODC expression did not show any other significant correlation. No statistically significant difference in relation to smoking or any other habits were observed in the expression of ARG1 and ODC, suggesting the altered expression is due to the diseased condition of the patients.

\section{Discussion}

We demonstrated, using both immunocytochemistry, and RT-PCR analysis, that blood of HNSCC patients has relatively frequent expression of ARG1 and ODC. We also analyzed their expression by immunohistochemical methods in a series of HNSCC tissues. To our knowledge, this is the first report of the status of the arginine metabolic enzymes expression in HNSCC patients. Presence of at least two distinct genes for ARG in mammals has been established-ARG1 strongly expressed in liver and red blood cells (RBCs), and ARG2 expressed in extrahepatic tissues. It is important to mention that mature RBCs are enucleated and they remain in circulation for 120 days. Although, the expression of much of the genome is repressed, yet a few hundred genes whose protein products are critical for viability and normal function of mature erythrocytes continue to be expressed till the last stage of terminal maturation (Benz et al., 2010). In human blood, neutrophiles and erythrocytes represent predominant ARG1 expressing population (Yang et al., 2013). Due to diseased condition, in HNSCC patients newly synthesized RBCs might have higher expression of ARG1 and ODC as evident by immunocytochemistry. Human ARG1 is also secreted in the microenvironment, in contrast to other mammalian ARG1, which confined within the cell (Munder et al., 2005; Jacobsen et al., 2007). Only one isoform ARG1 was present in blood cells, which probably plays role in polyamine biosynthesis (Stabellini et al., 2003) showing higher ODC activity in erythrocytes. This was further supported by the observation that OAT activity was undetectable in blood cells. In the studies conducted on serum obtained from 50 patients, we demonstrated that the mean preoperative ARG1 mRNA levels were significantly higher than from 100 healthy blood donors. The raised mRNA level was observed in all HNSCC patients. No difference in ARG1 expressions with smoking $(\mathrm{p}=0.15)$ was observed, therefore the altered expression was due to the carcinoma $(\mathrm{p}<0.0001)$. Any staining of ARG 2 in blood cells were considered false positive as we did not observe any ARG2 mRNA, and also support the recent study showing absence of ARG2 in blood (Yang et al., 2013). Although we observed OAT mRNA expression in blood with no detectable difference in OAT protein or mRNA expression, which might be circulating cell free mRNA (Garcia et al., 2008). We established the presence of both the isoforms of ARG in human head and neck squamous cell carcinoma tissues. ARG1 can also mediate immunoregulatory functions, in addition to its role in amino acid metabolism. ARG1 compete with nitric oxide synthase for L-arginine utilization, and subsequently suppress immune system of the patients by inhibiting NO production (Chang et al., 1998). T-lymphocytes play an important role in cell mediated immunity; rely on their microenvironment for the supply of L-arginine. With the appearance of ARG, decreased $\mathrm{T}$ cell proliferation, low expression of specific $\mathrm{T}$ cell receptors and decreased production of cytokines have been observed (Mussai et al., 2013). Perioperative arginine-enriched nutrition significantly improved the long-term overall survival and long-term disease-specific survival in malnourished patients with head and neck cancer (Buijs et al., 2010). Higher expression and activity of ARG1 are often observed in the blood of cancer patients (Polat et al., 2003; Grabon et al., 2009; Rodriguez et al., 2009). After surgical removal of malignant tumors, remnant cells can proliferate. Many studies demonstrated the role of polyamines in tumor cell growth. ODC is the first rate limiting enzyme in the biosynthesis of polyamines, which are essential for cell proliferation and regeneration (Mohammed et al., 2014). In several cancers including pancreas, esophageal lesions, elevated ODC levels have been reported and also linked to recurrence (Love et al., 2003). Tumor growth reduction occurs in rodent cancer models and cell lines treated with ODC-inhibiting substances (O'Brian et al., 1997). ARG inhibition has high therapeutic value in disorders due to impaired NO production. ARG isoforms also regulate the availability of proline for cell proliferation and collagen deposition during diseases such as asthma and cancer (Das et al., 2010). ARG1 and ODC enzymes are tightly regulated in normal tissue at the mRNA and protein levels and are deregulated in papillomas and carcinomas (Gilmour et al., 1987). Since polyamines and proline are vital for cell proliferation, increased ARG1 and ODC activity in blood and tissues may be linked to the development and progression of HNSCC.

In conclusion, upregulation of ARG1 and ODC expression occurs in HNSCC as compared with matching non-tumor blood and tissue, suggesting that these are the effects associated with oncogenic changes. Therefore, ARG1 and ODC gene and protein expression analysis could be useful as a biomarker for the determination of tumor biology. Higher ARG1 expression could also be described as an immunosuppressive mechanism. Further studies of HNSCC are warranted to confirm these results in larger patient populations as well as to explore the impact of ARG and ODC expression in tumor biology.

\section{Acknowledgements}

The study was financially supported by Department of Biotechnology, Government of India. S.S. was supported by DBT Research Associateship. The authors would like to thank Dr. Biswadeep Choudhury and Dr. Sumita Duttagupta, Silchar Medical College and Hospital, Silchar for providing HNSCC samples. 


\section{References}

Bhattacharjee A, Chakraborty A, Purkaystha P (2006). Prevalence of head and neck cancers in the north east - an institutional study. Indian J Otolaryngol Head Neck Surg, 58, 15-9.

Bergeron C, Boulet LP, Page N, et al (2007). Influence of cigarette smoke on the arginine pathway in asthmatic airways: increased expression of arginase I. J Allergy Clin Immunol, 119, 391-7.

Boffetta P, Hecht S, Gray N, et al (2008). Smokeless tobacco and cancer. Lancet Oncol, 9, 667-75.

Benz EJ Jr (2010). Learning about genomics and disease from the anucleate human red blood cell. J Clin Invest, 120, 4204-6.

Biczo G, Hegyi P, Berczi S, et al (2010). Inhibition of arginase activity ameliorates L-arginine-induced acute pancreatitis in rats. Pancreas, 39, 868-74.

Buijs N, van Bokhorst-de van der Schueren MA, Langius JA, et al (2010). Perioperative arginine-supplemented nutrition in malnourished patients with head and neck cancer improves long-term survival. Am J Clin Nutr, 92, 1151-6.

Barron L, Smith AM, El Kasmi KC, et al (2013). Role of arginase 1 from myeloid cells in th2-dominated lung inflammation. PLoS One, 8, 61961.

Chang CI, Liao JC, Kuo L (1998). Arginase modulates nitric oxide production in activated macrophages. Am J Physiol, 274, 342-8

Choudhury B, Srivastava S, Choudhury HH, et al (2014). Arginase and C-reactive protein as potential serum-based biomarker of head and neck squamous cell carcinoma patients of north east India. Tumor Biol, 35, 6739-48.

Das P, Lahiri A, Chakravortty D (2010). Modulation of the arginase pathway in the context of microbial pathogenesis: a metabolic enzyme moonlighting as an immune modulator. PLoS Pathog, 6, 1000899.

Dal Maso L, Torelli N, Biancotto E, et al (2015). Combined effect of tobacco smoking and alcohol drinking in the risk of head and neck cancers: a re-analysis of case-control studies using bi-dimensional spline models. Eur J Epidemiol, [Epub ahead of print]

ErberWN,Pinching AJ,Mason DY,(1984).Immunohistochemical detection of $\mathrm{T}$ and $\mathrm{B}$ cell populations in routine blood smears. Lancet, 323, 1042-6.

Gilmour SK, Verma AK, Madara T, et al (1987). Regulation of ornithine decarboxylase gene expression in mouse epidermis and epidermal tumors during two-stage tumorigenesis. Cancer Res, 47, 1221-5.

Gijare PS, Rao KVK, Bhide SV, (1989). Effects of tobaccospecific nitrosamines and snuff extract on cell proliferation and activities of ornithine decarboxylase and aryl hydrocarbon hydroxylase in mouse tongue primary epithelial cell cultures. $J$ Can Res Clin Oncol, 115, 558-63.

Gourin CG, Podolsky RH, (2006). Racial disparities in patients with head and neck squamous cell carcinoma. Laryngoscope, 116, 1093-106.

Garcia V, Garcia JM, Pena C, et al (2008). Free circulating mRNA in plasma from breast cancer patients and clinical outcome. Cancer Lett, 263, 312-20.

Grabon W, Mielczarek-Puta M, Chrzanowska A, et al (2009). $\mathrm{L}$-arginine as a factor increasing arginase significance in diagnosis of primary and metastatic colorectal cancer. Clin Biochem, 42, 353-7.

Haenisch B, von Kugelgen I, Bonisch H, et al (2008). Regulatory mechanisms underlying agmatine homeostasis in humans. Am J Physiol Gastrointest Liver Physiol, 295, 1104-10

Hashibe M, Brennan P, Chuang SC, et al (2009) Interaction between tobacco and alcohol use and the risk of head and neck cancer: pooled analysis in the International Head and Neck Cancer Epidemiology Consortium. Can Epidem Biomark Prev, 18, 541-50.

Hauswald H, Simon C, Hecht S, et al (2011). Long-term outcome and patterns of failure in patients with advanced head and neck cancer. Radiat Oncol, 6, 70.

Jacobsen LC, Theilgaard-Monch K, Christensen EI, et al (2007). Arginase 1 is expressed in myelocytes/metamyelocytes and localized in gelatinase granules of human neutrophils. Blood, 109, 3084-7.

Kitowska K, Zakrzewicz D, Konigshoff M, et al (2008). Functional role and species-specific contribution of arginases in pulmonary fibrosis. Am J Physiol Lung Cell Mol Physiol, 294, 34-45.

Kim J, Shin JM, Jeon YJ, et al (2012). Proteomic validation of multifunctional molecules in mesenchymal stem cells derived from human bone marrow, umbilical cord blood and peripheral blood. PLoS One, 7, 32350 .

Love RR, Astrow SH, Cheeks AM, et al (2003). Ornithine decarboxylase (ODC) as a prognostic factor in operable breast cancer. Breast Cancer Res Treat, 79, 329-34.

Lopez V, Alarcon R, Orellana MS, et al (2005). Insights into the interaction of human arginase II with substrate and manganese ions by site-directed mutagenesis and kinetic studies. Alteration of substrate specificity by replacement of Asn 149 with Asp. Febs J, 272, 4540-8.

Matthaei KI, Williams JF (1987). Ornithine aminotransferase turnover in host tissues of tumor-bearing rats. J Nat Can Inst, 79, 805-9.

Molnar B, Ladanyi A, Tanko L, et al (2001). Circulating tumor cell clusters in the peripheral blood of colorectal cancer patients. Clin Can Res, 7, 4080-5.

Munder M, Mollinedo F, Calafat J, et al (2005). Arginase I is constitutively expressed in human granulocytes and participates in fungicidal activity. Blood, 105, 2549-56.

Mondal R, Ghosh SK, Choudhury JH, et al (2013). Mitochondrial DNA copy number and risk of oral cancer: a report from Northeast India. PLoS One, 8, 57771.

Mussai F1,De Santo C,Abu-Dayyeh I, et al (2013). Acute myeloid leukemia creates an arginase-dependent immunosuppressive microenvironment. Blood, 122, 749-58.

Mohammed A, Janakiram NB, Madka V, et al (2014). Eflornithine (DFMO) prevents progression of pancreatic cancer by modulating ornithine decarboxylase signaling. Cancer Prev Res (Phila), 7, 1198-209.

O'Brian TG, Megosh LC, et al (1997). Ornithine decarboxylase over expression is a sufficient condition for tumor promotion in mouse skin. Cancer Res, 57, 2630-7.

Polat MF, Taysİ S, Polat S, et al (2003). Elevated serum arginase activity levels in patients with breast cancer. Surg Today, 33, 655-61.

Rodriguez PC, Ernstoff MS, Hernandez C, et al (2009). Arginase I-producing myeloid-derived suppressor cells in renal cell carcinoma are a subpopulation of activated granulocytes. Can Res, 69, 1553-60.

Ramirez-Zamora S, Mendez-Rodriguez ML, Olguin-Martinez $M$, et al (2013). Increased erythrocytes by-products of arginine catabolism are associated with hyperglycemia and could be involved in the pathogenesis of type 2 diabetes mellitus. PLoS One, 8, 66823.

Stabellini G, Calastrini C, Gagliano N, et al (2003). Polyamine levels and ornithine decarboxylase activity in blood and erythrocytes in human diseases. Int J Clin Pharmacol Res, 23, 17-22.

Skorupski KA, O’Brien TG, Guerrero T, et al (2011). Phase I/II clinical trial of 2-difluoromethyl-ornithine (DFMO) and a 
Shilpee Srivastava and Sankar Kumar Ghosh

novel polyamine transport inhibitor (MQT 1426) for feline oral squamous cell carcinoma. Vet Comp Oncol, 9, 275-82.

Vilas-Boas W, Cerqueira BA, Zanette AM, et al (2010). Arginase levels and their association with Th17-related cytokines, soluble adhesion molecules (sICAM-1 and SVCAM-1) and hemolysis markers among steady-state sickle cell anemia patients. Ann Hematol, 89, 877-82.

Wolf C, Bruss M, Hanisch B, et al (2007). Molecular basis for the antiproliferative effect of agmatine in tumor cells of colonic, hepatic, and neuronal origin. Mol Pharmacol, 71, 276-83

Yamamoto Y, Sousse LE, Enkhbaatar P, et al (2012). Gammatocopherol nebulization decreases oxidative stress, arginase activity, and collagen deposition after burn and smoke inhalation in the ovine model. Shock, 38, 671-6.

Yang J, Gonon AT, Sjoquist PO, et al (2013). Arginase regulates red blood cell nitric oxide synthase and export of cardioprotective nitric oxide bioactivity. Proc Natl Acad Sci, 110, 15049-54. 